\title{
Implementing Multiparty Session Types in Rust
}

\author{
Nicolas Lagaillardie ${ }^{1(\otimes)}(\mathbb{D})$, Rumyana Neykova ${ }^{2(\bowtie)}(\mathbb{D}$, \\ and Nobuko Yoshida ${ }^{1(\otimes)}(\mathbb{D}$ \\ 1 Imperial College London, London, UK \\ \{n.lagaillardie19,n.yoshida\}@imperial.ac.uk \\ 2 Brunel University London, London, UK \\ rumyana.neykova@brunel.ac.uk
}

\begin{abstract}
Multiparty Session Types (MPST) is a typing discipline for distributed protocols, which ensures communication safety and deadlockfreedom for more than two participants. This paper reports on our research project, implementing multiparty session types in Rust. Current Rust implementations of session types are limited to binary (twoparty communications). We extend an existing library for binary session types to MPST. We have implemented a simplified Amazon Prime Video Streaming protocol using our library for both shared and distributed communication transports.
\end{abstract}

\section{Introduction}

In the last decade, the software industry has seen a shift towards programming languages that promote the coordination of concurrent and/or distributed software components through the exchange of messages over communication channels. Languages with native message-passing primitives (e.g., Go, Elixir and Rust) are becoming increasingly popular. In particular, Rust has been named the most loved programming language in the annual Stack Overflow survey for four consecutive years $(2016-19)^{1}$.

The advantage of message-passing concurrency is well-understood: it allows cheap horizontal scalability at a time when technology providers have to adapt and scale their tools and applications to various devices and platforms. Messagepassing based software, however, is as vulnerable to errors as other concurrent programming techniques [16]. Much academic research has been done to develop rigorous theoretical frameworks for verification of message-passing programs. One such framework is multiparty session types (MPST) [5] - a type-based discipline that ensures that concurrent and distributed systems are safe by design. It guarantees that message-passing processes following a predefined communication protocol, are free from communication errors and deadlocks.

\footnotetext{
1 https://insights.stackoverflow.com/survey/2019.

(C) IFIP International Federation for Information Processing 2020

Published by Springer Nature Switzerland AG 2020

S. Bliudze and L. Bocchi (Eds.): COORDINATION 2020, LNCS 12134, pp. 127-136, 2020.

https://doi.org/10.1007/978-3-030-50029-0_8
} 
Rust is a particularly appealing language for the practical embedding of session types. Its affine type system allows for static typing of linear resources an essential requirement for the safety of session type systems. Rust combines efficiency with message-passing abstractions, thread and memory safety [15], and has been used for the implementation of large-scale concurrent applications such as the Mozilla browser, Firefox, and the Facebook blockchain platform, Libra. Despite the interest in the Rust community for verification techniques handling multiple communicating processes ${ }^{2}$, the existing Rust implementations [8,9] are limited to binary (two-party) session types.

In this short paper, we present our design and implementation for multiparty session types in Rust. Our design follows a state-of-the-art encoding of multiparty into binary session types [13]. We generate local types in Rust, utilising the Scribble toolchain $[12,18]$. Our library for MPST programming in Rust, mpst-rust, is implemented as a thin wrapper over an existing binary session types library [9]. Differently from other MPST implementations that check the linear usage of channels at runtime (e.g. [6,13]), we rely on the Rust affine type system to type-check MPST programs. In addition, since we generate the local types from a readable global specification, errors caused by an affine (and not linear) usage of channels, a well-known limitation of the previous libraries $[8,9]$, are easily avoided.

This paper is organised as follows: Sect. 2 gives an overview of our framework with a usecase; Sect. 3 shows our implementation and discusses the advantages of our approach; and Sect. 4 concludes with related and future work. Our library is available from https://github.com/NicolasLagaillardie/mpst_rust_github.

\section{From Binary to Multiparty Sessions in Rust}

Framework Overview: MPST in Rust. Our design resembles the top-down methodology of multiparty session types, as illustrated in Fig. 1. It follows three main steps [5,17]. First, a global type, also called a global protocol, is defined as a shared contract between communicating endpoint processes. A global protocol is then projected to each endpoint process, resulting in a local type. A local type involves only the interactions specific to a given endpoint. Finally, each endpoint process is type-checked against its projected local type.

The specific parts of our framework that distinguish it from other stateof-the-art MPST works are highlighted in red, which corresponds to our new library for MPST programming in Rust, mpst-rust. It is realised as a thin wrapper on top of an existing Rust library for validation of binary (2-party-only) session types. Developers use the MPST primitives provided by mpst-rust to implement endpoint programs. Also, our framework allows the types for each communication primitive to be either (1) generated from the Scribble toolchain; or (2) written by the developers. The Scribble toolchain [18] provides facilities for writing, verifying and projecting global protocols. Our framework guarantees

\footnotetext{
${ }^{2}$ https://users.rust-lang.org/t/anybody-working-on-multiparty-session-types-forrust/10610.
} 

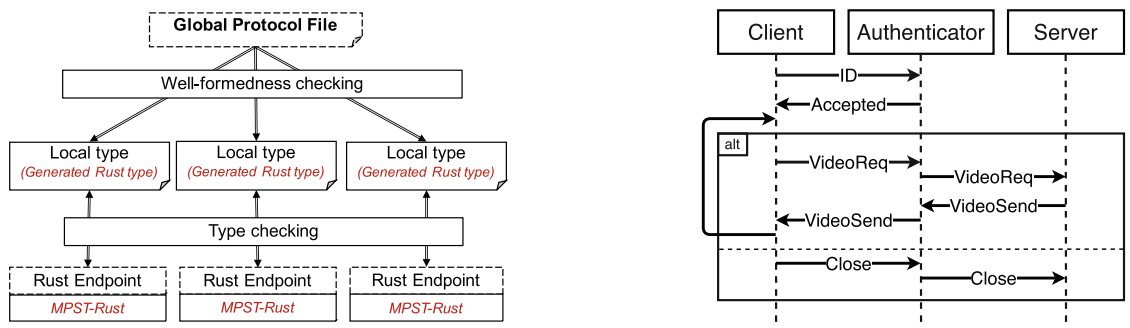

Fig. 1. MPST Workflow (left) and Amazon Prime Video usecase (right)

that processes implemented using mpst-rust primitives with Scribble-generated types are free from deadlocks, reception errors, and protocol deviations. Next, we explain, via an example, how the framework of MPST can be applied to Rust.

Example: Amazon Prime Video Streaming. The Amazon Prime Video streaming service is a usecase which can take full advantage of multiparty session types. Each streaming application connects to servers, and possibly other devices, to access services, and follows some specific protocol. To present our design, we use a simplified version of the protocol, illustrated in the diagram in Fig. 1 (right). The diagram should be read from top to bottom. The protocol involves three services - an Authenticator service, a Server and a Client. At first, Client connects to Authenticator by providing an identifying id. If the id is accepted, the session continues with a choice on Client to either request a video or end the session. The first branch is, a priori, the main service provided by Amazon Prime Video. Client cannot directly request videos from Server, and has to go through Authenticator instead. On the diagram, the choice is denoted as the frame alt and the choices are separated with the horizontal dotted line. The protocol is recursive, and $\mathrm{Client}$ can request new videos as many times as needed. The arrow going back on Client side in Fig. 1 represents this recursive behaviour. To end the session, Client first sends Close message to Authenticator, which then subsequently sends a Close message to Server.

Implementing the Authenticator role Using mpst-rust. Due to space limitations, we only show the implementation of the Authenticator role (hereafter role A), the implementations of the other roles (role B for the Server and role C for the Client) are similar. The Rust code for role A using the mpst-rust library is given in Fig. 2 (left). It closely follows the local protocol in Fig. 2 (right), that is projected from the global protocol by the Scribble toolchain. First, line 1 declares a function authenticator that is parametric in a multiparty channel s of type VideoP_A. The type VideoP_A specifies which operations are allowed on s. This type can either be written by the developer, or generated by Scribble (cf. Listing 1).

On line 3, A receives an identifying id from $\mathrm{C}$. The function recv_mpst_a_to_c, provided by mpst-rust library returns the received value (the id) and the new multiparty channel, to be used in subsequent communications. Line 3 rebinds 
the protocol is QueueAVideo. Note that, according to the protocol, A first has to receive a VideoRequest message from $\mathrm{C}$, and then it has to forward that message to B Hence, swapping of lines 17 and 18 from Fig. 2 is a protocol violation error. We can detect such violations since the queue for the type ChoiceA: :Video, QueueAVideo (line 21), is specified as RoleAtoC<RoleAtoB ...>, which codifies that first the channel for $\mathrm{C}$ and then the channel for $\mathrm{B}$ should be used. Note that none of the defined queues is recursive. Recursion is implicitly specified on binary types, while each queue is related to a SessionMpst type.

Distributed Execution Environment. The default transport of mpst-rust is the built-in Rust communication channels (crossbeam_channel). Also, to test our example in a more realistic distributed environment, we have also connected each process through MQTT (MQ Telemetry Transport) [7]. MQTT is a messaging middleware for exchanging messages between devices, predominantly used in IoT networks. At the start of the protocol, each process connects to a public MQTT channel, and a session is established. Therefore, we have mapped binary channels to MQTT sockets, in addition to the built-in Rust channels.

\section{Design and Implementation of mpst-rust}

Multiparty Channels as an Ordered Tuple of Binary Channels. The main idea of the design of our framework is that a multiparty session can be realised with two ingredients: (1) a list of separate binary sessions (one session for each pair of participants) and (2) a queue that imposes the ordering between the binary channels. Listing 2 (lines 2-3) shows the implementation of a multiparty channel in a 3-party protocol. The SessionMpst structure holds two fields, session 1 and session2, that are of a binary session type. For an illustration purpose, we show only the implementation of a multiparty channel for three processes. The same approach can be generalised, using our code generation tool, to any number of communicating processes. For example, in case of a protocol with four roles, each multiparty session will have four fields - a field for the binary session between each pair of participants and a field for the queue.

The order of usage of the binary channels of a SessionMpst object is stored inside the queue field. For instance, the behaviour that role A has to communicate first with role $B$, then with a role $\mathrm{C}$, and then the session ends can be specified using a queue of type RoleAtoB<RoleAtoC<RoleEnd $>$. Note that all queue types, such as RoleAtoB, RoleAtoC, are generated.

MPST Communication Primitives as Wrappers of Binary Channels. As explained in Sect.2, programming with mpst-rust relies on communication primitives, such as send_mpst_a_to_b, that have the sender and receiver roles baked into their name. To ensure that the binary channels are used as specified by the global protocol, each communication function is parametric on a generic quadruple type $<\mathrm{T}, \mathrm{S} 1, \mathrm{~S} 2, \mathrm{R}>$ where $\mathrm{T}$ is a payload type, $\mathrm{S} 1$ and $\mathrm{S} 2$ are binary session types and $\mathrm{R}$ is a type for a queue (MPST-queue type) that imposes the order in which the binary sessions inside a multiparty session must be used. 


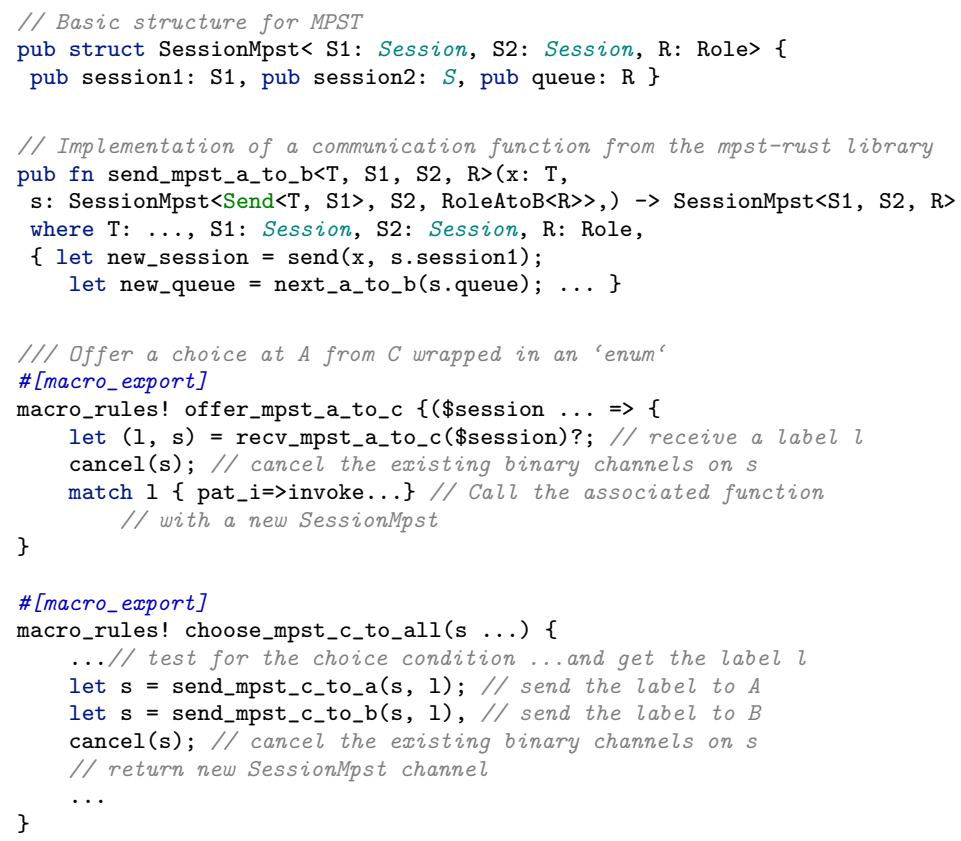

Listing 2. MPST Rust communication primitives

Listing 2 (lines 5-9) shows the implementation for send_mpst_a_to_b(). As clear from the type parameters, the client of the function should supply a MPSTqueue type RoleAtoB $<\mathrm{R}>$. The binary session type $\mathrm{S} 1$ should be encapsulated in a Send $<\mathrm{T}, \mathrm{S} 1>$. The body of the function sends the message of type $\mathrm{T}$ on the binary channel stored in the first field, session1 (corresponding to the binary session with role B), of the multiparty session s. Since the communication is on a binary channel, we reuse the binary send primitive from [9].

External and internal choices are implemented as macros that require an argument of type SessionMpst. The implementation of offer_mpst_a_to_c is given in lines 11-14. In essence, a choice is implemented as a broadcast from one role to the others. In our usecase, the active role that makes the choice is C. Hence, the macro offer_mpst_a_to_c explicitly performs a receive (recv_mpst_a_to_c $(s))$ on the session channel $s$. The received value is pattern matched and passed to any of the functions given as arguments to offer_mpst_a_to_c. Similarly, choose_mpst_c_to_all in lines 19-26 is a macro that performs a select operation. The active role $\mathrm{C}$ sends the selected label to all roles in the protocol. In our particular example, $\mathrm{C}$ sends the selected label 1 to $\mathrm{A}$ and $\mathrm{B}$.

Discussions. Our implementation, although intuitive, does not resolve the inherent conflict between Rust, which is affine, and session types, which are linear. The implementation suffers from the same drawback as [9]. However, the MPST methodology is a step forward in terms of usability. Differently than the Rust local types which can get convoluted, the syntax of global protocols 
is user-friendly and readable. Developers can use the global protocol as guidance, and hence avoid errors such as prematurely ending of a session. Moreover, as observed in Kokke's library [9], most of the errors are caused by misuse of methods and functions. Since we are code-generating the local types, the chance of misspelling is significantly reduced. Another viable option for our framework is to take the bottom-up approach: to check directly whether a set of manuallywritten Rust local types satisfy safety/liveness properties by a model checker [14] or the multiparty compatibility (a property which guarantees deadlock-freedom of communicating automata, which are equivalent to local session types) $[2,11]$.

\section{Related and Future Work}

The Rust library in [8] implements binary session types, following [4]. It checks at compile-time that the behaviours of two endpoint processes are dual, i.e the processes are compatible. The library in [9], based on the EGV calculus by Fowler et al. [3], provides constructs for writing and checking binary session types, and additionally supports exception handling constructs. We build on top of the library in [9] since it offers several improvements in comparison to [8]. Most importantly, the treatment of closing a channel prematurely in [8] may lead to memory leaks. Both libraries suffer from a well-known limitation of binary session types ${ }^{3}$. Notably, since deadlock-freedom is ensured only inside a session, a Rust endpoint process, that communicates with more than one other process, is prone to deadlocks and communication errors. Our framework solves that limitation by expanding the scope of a session to multiple participants.

Our proposed design follows the methodology given by [6], which generates Java communicating APIs from Scribble. This, and other multiparty session types implementations, exploit the equivalence between local session types and communicating automata to generate session types APIs for mainstream programming languages (e.g., Java [6,10], Go [1], F\# [13]). Each state from state automata is implemented as a class, or in the case of [10], as a type state. To ensure safety, state automata have to be derived from the same global specification. All of the works in this category use the Scribble toolchain to generate the state classes from a global specification and detect linearity violations at runtime. This paper proposes the generation of protocol-specific APIs, which promotes type checking of protocols at compile-time. This is done by projecting the endpoints' state space in those protocols to groups of channel types in the desired language. In the future, we plan to implement the bottom-up approach, in addition to the top-down approach outlined in this paper, as to compare their productivity and scalability.

Acknowledgement. The work has been partially supported by the following funding schemes VeTSS, EPSRC EP/K011715/1, EP/K034413/1, EP/L00058X/1, $\mathrm{EP} / \mathrm{N} 027833 / 1, \mathrm{EP} / \mathrm{N} 028201 / 1, \mathrm{EP} / \mathrm{T} 006544 / 1$ and, EP/T014709/1.

$\overline{3}$ https://github.com/Munksgaard/session-types/issues/62. 


\section{References}

1. Castro, D., Hu, R., Jongmans, S.S., Ng, N., Yoshida, N.: Distributed programming using role parametric session types in Go. In: 46th ACM SIGPLAN Symposium on Principles of Programming Languages, vol. 3, pp. 29:1-29:30. ACM (2019)

2. Deniélou, P.-M., Yoshida, N.: Multiparty compatibility in communicating automata: characterisation and synthesis of global session types. In: Fomin, F.V., Freivalds, R., Kwiatkowska, M., Peleg, D. (eds.) ICALP 2013. LNCS, vol. 7966, pp. 174-186. Springer, Heidelberg (2013). https://doi.org/10.1007/978-3-642-39212-2_ 18

3. Fowler, S., Lindley, S., Morris, J.G., Decova, S.: Exceptional asynchronous session types: session types without tiers. Proc. ACM Program. Lang. 3(POPL), 28:128:29 (2019). https://doi.org/10.1145/3290341

4. Honda, K., Vasconcelos, V.T., Kubo, M.: Language primitives and type discipline for structured communication-based programming. In: Hankin, C. (ed.) ESOP 1998. LNCS, vol. 1381, pp. 122-138. Springer, Heidelberg (1998). https://doi.org/ 10.1007/BFb0053567

5. Honda, K., Yoshida, N., Carbone, M.: Multiparty asynchronous session types. POPL 43(1), 273-284 (2008)

6. Hu, R., Yoshida, N.: Hybrid session verification through endpoint API generation. In: Stevens, P., Wąsowski, A. (eds.) FASE 2016. LNCS, vol. 9633, pp. 401-418. Springer, Heidelberg (2016). https://doi.org/10.1007/978-3-662-49665-7_24

7. Hunkeler, U., Truong, H.L., Stanford-Clark, A.: MQTT-S-a publish/subscribe protocol for wireless sensor networks. In: 2008 3rd International Conference on Communication Systems Software and Middleware and Workshops, COMSWARE 2008, pp. 791-798. IEEE (2008)

8. Jespersen, T.B.L., Munksgaard, P., Larsen, K.F.: Session types for Rust. In: Proceedings of the 11th ACM SIGPLAN Workshop on Generic Programming, pp. 13-22. ACM (2015). https://doi.org/10.1145/2808098.2808100

9. Kokke, W.: Rusty variation: deadlock-free sessions with failure in Rust. In: Proceedings 12th Interaction and Concurrency Experience, ICE 2019, Copenhagen, Denmark, 20-21 June 2019, pp. 48-60 (2019). https://doi.org/10.4204/EPTCS. 304.4

10. Kouzapas, D., Dardha, O., Perera, R., Gay, S.J.: Typechecking protocols with Mungo and StMungo. In: PPDP, pp. 146-159 (2016). https://doi.org/10.1145/ 2967973.2968595

11. Lange, J., Yoshida, N.: Verifying asynchronous interactions via communicating session automata. In: Dillig, I., Tasiran, S. (eds.) CAV 2019. LNCS, vol. 11561, pp. 97-117. Springer, Cham (2019). https://doi.org/10.1007/978-3-030-25540-4_6

12. Jespersen, T.B.L., Munksgaard, P., Larsen, K.F.: Session types for Rust. In: Proceedings of the 11th ACM SIGPLAN Workshop on Generic Programming, pp. 13-22. Association for Computing Machinery, New York (2015). https://doi.org/ 10.1145/2808098.2808100. ISBN 9781450338103

13. Scalas, A., Dardha, O., Hu, R., Yoshida, N.: A linear decomposition of multiparty sessions for safe distributed programming. In: 31st European Conference on ObjectOriented Programming. LIPIcs, vol. 74, pp. 24:1-24:31. Schloss Dagstuhl (2017)

14. Scalas, A., Yoshida, N.: Less is more: multiparty session types revisited. In: 46th ACM SIGPLAN Symposium on Principles of Programming Languages, vol. 3, pp. 30:1-30:29. ACM (2019) 
15. Klabnik, S., Nichols, C.: The Rust Programming Language. 1.35.0 edn. (2019). https://doc.rust-lang.org/1.35.0/book/. Contributions from the Rust Community

16. Tu, T., Liu, X., Song, L., Zhang, Y.: Understanding real-world concurrency bugs in Go. In: ASPLOS, pp. 865-878. ACM (2019)

17. Yoshida, N., Gheri, L.: A very gentle introduction to multiparty session types. In: Hung, D.V., D'Souza, M. (eds.) ICDCIT 2020. LNCS, vol. 11969, pp. 73-93. Springer, Cham (2020). https://doi.org/10.1007/978-3-030-36987-3_5

18. Yoshida, N., Hu, R., Neykova, R., Ng, N.: The scribble protocol language. In: Abadi, M., Lluch Lafuente, A. (eds.) TGC 2013. LNCS, vol. 8358, pp. 22-41. Springer, Cham (2014). https://doi.org/10.1007/978-3-319-05119-2_3 